\title{
A sequence of Albin type continuous martingales with Brownian marginals and scaling
}

D. Baker ${ }^{1}$, C. Donati-Martin ${ }^{1}$, and M. Yor ${ }^{12}$

1 UPMC Univ Paris 06 and CNRS, UMR 7599, Laboratoire de Probabilités et Modèles Aléatoires, 4, place Jussieu, 75252 Paris cedex 05.

E-mail:david.baker@etu.upmc.fr, catherine.donati@upmc.fr

${ }^{2}$ Institut Universitaire de France

Dedicated to Lester Dubins (1921-2010) to whom the third author owes a lot.

Summary. Closely inspired by Albin's method which relies ultimately on the duplication formula for the Gamma function, we exploit Gauss' multiplication formula to construct a sequence of continuous martingales with Brownian marginals and scaling.

Key words: Martingales, Brownian marginals

\section{Motivation and main results}

(1.1) Knowing the law of a "real world" random phenomena, i.e. random process, $\left(X_{t}, t \geq 0\right)$ is often extremely difficult and in most instances, one avails only of the knowledge of the 1-dimensional marginals of $\left(X_{t}, t \geq 0\right)$. However, there may be many different processes with the same given 1-dimensional marginals.

In the present paper, we make explicit a sequence of continuous martingales $\left(M_{m}(t), t \geq 0\right)$ indexed by $m \in \mathbb{N}$ such that for each $m$,

i) $\left(M_{m}(t), t \geq 0\right)$ enjoys the Brownian scaling property: for any $c>0$,

$$
\left(M_{m}\left(c^{2} t\right), t \geq 0\right) \stackrel{(l a w)}{=}\left(c M_{m}(t), t \geq 0\right)
$$

ii) $M_{m}(1)$ is standard Gaussian.

Note that, combining i) and ii), we get, for any $t>0$

$$
M_{m}(t) \stackrel{(l a w)}{=} B_{t},
$$


where $\left(B_{t}, t \geq 0\right)$ is a Brownian motion, i.e. $M_{m}$ admits the same 1dimensional marginals as Brownian motion.

(1.2) Our main result is the following extension of Albin's construction [1] from $m=1$ to any integer $m$.

Theorem 1. Let $m \in \mathbb{N}$. Then, there exists a continuous martingale $\left(M_{m}(t), t \geq\right.$ 0) which enjoys i) and ii) and is defined as follows:

$$
M_{m}(t)=X_{t}^{(1)} \ldots X_{t}^{(m+1)} Z_{m}
$$

where $\left(X_{t}^{(i)}, t \geq 0\right)$, for $i=1, \ldots, m+1$, are independent copies of the solution of the $S D E$

$$
d X_{t}=\frac{1}{m+1} \frac{d B_{t}}{X_{t}^{m}} ; \quad X_{0}=0
$$

and, furthermore, $Z_{m}$ is independent from $\left(X^{(1)}, \ldots, X^{(m+1)}\right)$ and

$$
Z_{m} \stackrel{(\text { law })}{=}(m+1)^{1 / 2}\left(\prod_{j=0}^{m-1} \beta\left(\frac{1+2 j}{2(m+1)}, \frac{m-j}{m+1}\right)\right)^{\frac{1}{2(m+1)}}
$$

where $\beta(a, b)$ denotes a beta variable with parameter $(a, b)$ with density

$$
\frac{\Gamma(a+b)}{\Gamma(a) \Gamma(b)} x^{a-1}(1-x)^{b-1} 1_{[0,1]}(x)
$$

and the beta variables on the right-hand side of (3) are independent.

Remark: For $m=1, Z_{1}=\sqrt{2}\left(\beta\left(\frac{1}{4}, \frac{1}{2}\right)\right)^{1 / 4}$ and we recover the distribution of $Y:=Z_{1}$ given by $(2)$ in [1].

(1.3) For the convenience of the reader, we also recall that, if one drops the continuity assumption when searching for martingales $(M(t) ; t \geq 0)$ satisfying i) and ii), then, the Madan-Yor construction [5] based on the "Azéma-Yor under scaling" method provides such a martingale.

Precisely, starting from a Brownian motion $\left(B_{u}, u \geq 0\right)$ and denoting $S_{u}=$ $\sup _{s \leq u} B_{s}$, introduce the family of stopping times

$$
\tau_{t}=\inf \left\{u, S_{u} \geq \psi_{t}\left(B_{u}\right)\right\}
$$

where $\psi_{t}$ denotes the Hardy-Littlewood function associated with the centered Gaussian distribution $\mu_{t}$ with variance $t$, i.e.

$$
\begin{aligned}
\psi_{t}(x) & =\frac{1}{\mu_{t}([x, \infty[)} \int_{x}^{\infty} y \exp \left(-\frac{y^{2}}{2 t}\right) \frac{d y}{\sqrt{2 \pi t}} \\
& =\sqrt{t} \exp \left(-\frac{x^{2}}{2 t}\right) / \mathcal{N}(x / \sqrt{t})
\end{aligned}
$$

where $\mathcal{N}(a)=\int_{a}^{\infty} \exp \left(-\frac{y^{2}}{2}\right) d y$. Then, $M_{t}=B_{\tau_{t}}$ is a martingale with Brownian marginals.

Another solution has been given by Hamza and Klebaner [4]. 


\section{Proof of the theorem}

Step 1: For $m \in \mathbb{R}$ and $c \in \mathbb{R}$, we consider the stochastic equation:

$$
d X_{t}=c \frac{d B_{t}}{X_{t}^{m}}, \quad X_{0}=0 .
$$

This equation has a unique weak solution which can be defined as a timechanged Brownian motion

$$
\left(X_{t}\right) \stackrel{(\text { law })}{=} W\left(\alpha^{(-1)}(t)\right)
$$

where $W$ is a Brownian motion starting from 0 and $\alpha^{(-1)}$ is the (continuous) inverse of the increasing process

$$
\alpha(t)=\frac{1}{c^{2}} \int_{0}^{t} W_{u}^{2 m} d u .
$$

We look for $k \in \mathbb{N}$ and $c$ such that $\left(X_{t}^{2 k}, t \geq 0\right)$ is a squared Bessel process of some dimension $d$. It turns out, by application of Itô's formula, that we need to take $k=m+1$ and $c=\frac{1}{m+1}$. Thus, we find that $\left(X_{t}^{2(m+1)}, t \geq 0\right)$ is a squared Bessel process with dimension $d=k(2 k-1) c^{2}=\frac{2 m+1}{m+1}$.

Note that the law of a $\operatorname{BESQ}(d)$ process at time 1 is well known to be that of $2 \gamma_{d / 2}$, where $\gamma_{a}$ denotes a gamma variable with parameter $a$. Thus, we have:

$$
\left|X_{1}\right| \stackrel{(\text { law })}{=}\left(2 \gamma_{\frac{2 m+1}{2(m+1)}}\right)^{\frac{1}{2(m+1)}}
$$

Step 2: We now discuss the scaling property of the solution of (2). From the scaling property of Brownian motion, it is easily shown that, for any $\lambda>0$, we get:

$$
\left(X_{\lambda t}, t \geq 0\right) \stackrel{(\text { law })}{=}\left(\lambda^{\alpha} X_{t}, t \geq 0\right)
$$

with $\alpha=\frac{1}{2(m+1)}$, that is, the process $\left(X_{t}, t \geq 0\right)$ enjoys the scaling property of order $\frac{1}{2(m+1)}$.

Step 3: Consequently, if we multiply $m+1$ independent copies of the process $\left(X_{t}, t \geq 0\right)$ solution of $(2)$, we get a process

$$
Y_{t}=X_{t}^{(1)} \ldots X_{t}^{(m+1)}
$$

which is a martingale and has the scaling property of order $\frac{1}{2}$.

Step 4: Finally, it suffices to find a random variable $Z_{m}$ independent of the processes $X_{t}^{(1)}, \ldots, X_{t}^{(m+1)}$ and which satisfies:

$$
N \stackrel{(\text { law })}{=} X_{1}^{(1)} \ldots X_{1}^{(m+1)} Z_{m}
$$


where $N$ denotes a standard Gaussian variable. Note that the distribution of any of the $X_{1}^{(i)}$ 's is symmetric. We shall take $Z_{m} \geq 0$; thus, the distribution of $Z_{m}$ shall be determined by its Mellin transform $\mathcal{M}(s)$. From $(5), \mathcal{M}(s)$ satisfies:

$$
\mathbb{E}\left[\left(2 \gamma_{1 / 2}\right)^{s / 2}\right]=\left(\mathbb{E}\left[\left(2 \gamma_{d / 2}\right)^{s / 2(m+1)}\right]\right)^{m+1} \mathcal{M}(s)
$$

with $d=\frac{2 m+1}{m+1}$, that is:

$$
2^{s / 2} \frac{\Gamma\left(\frac{1+s}{2}\right)}{\Gamma\left(\frac{1}{2}\right)}=2^{s / 2}\left(\frac{\Gamma\left(\frac{d}{2}+\frac{s}{2(m+1)}\right)}{\Gamma\left(\frac{d}{2}\right)}\right)^{m+1} \mathcal{M}(s)
$$

that is precisely:

$$
\frac{\Gamma\left(\frac{1+s}{2}\right)}{\Gamma\left(\frac{1}{2}\right)}=\left(\frac{\Gamma\left(\frac{2 m+1+s}{2(m+1)}\right)}{\Gamma\left(\frac{2 m+1}{2(m+1)}\right)}\right)^{m+1} \mathcal{M}(s) .
$$

Now, we recall Gauss multiplication formula ([2], see also [3])

$$
\Gamma(k z)=\frac{k^{k z-1 / 2}}{(2 \pi)^{\frac{k-1}{2}}} \prod_{j=0}^{k-1} \Gamma\left(z+\frac{j}{k}\right)
$$

which we apply with $k=m+1$ and $z=\frac{1+s}{2(m+1)}$. We then obtain, from (7)

$$
\begin{aligned}
\frac{\Gamma\left(\frac{1+s}{2}\right)}{\sqrt{\pi}} & =\frac{(m+1)^{s / 2}}{(2 \pi)^{m / 2}} \frac{1}{\sqrt{\pi}} \prod_{j=0}^{m} \Gamma\left(\frac{1+s+2 j}{2(m+1)}\right) \\
& =(m+1)^{s / 2} \prod_{j=0}^{m}\left(\frac{\Gamma\left(\frac{1+s+2 j}{2(m+1)}\right)}{\Gamma\left(\frac{1+2 j}{2(m+1)}\right)}\right)
\end{aligned}
$$

since the two sides of (8) are equal to 1 for $s=0$. We now plug (9) into (6) and obtain

$$
(m+1)^{s / 2} \prod_{j=0}^{m}\left(\frac{\Gamma\left(\frac{1+s+2 j}{2(m+1)}\right)}{\Gamma\left(\frac{1+2 j}{2(m+1)}\right)}\right)=\left(\frac{\Gamma\left(\frac{2 m+1+s}{2(m+1)}\right)}{\Gamma\left(\frac{2 m+1}{2(m+1)}\right)}\right)^{m+1} \mathcal{M}(s)
$$

We note that for $j=m$, the same term appears on both sides of (10), thus (10) may be written as:

$$
(m+1)^{s / 2} \prod_{j=0}^{m-1}\left(\frac{\Gamma\left(\frac{1+s+2 j}{2(m+1)}\right)}{\Gamma\left(\frac{1+2 j}{2(m+1)}\right)}\right)=\left(\frac{\Gamma\left(\frac{2 m+1+s}{2(m+1)}\right)}{\Gamma\left(\frac{2 m+1}{2(m+1)}\right)}\right)^{m} \mathcal{M}(s)
$$

In terms of independent gamma variables, the left-hand side of (11) equals: 


$$
(m+1)^{s / 2} \mathbb{E}\left[\left(\prod_{j=0}^{m-1} \gamma_{\frac{1+2 j}{2(m+1)}}^{(j)}\right)^{\frac{s}{2(m+1)}}\right]
$$

whereas the right-hand side of (11) equals:

$$
\mathbb{E}\left[\left(\prod_{j=0}^{m-1} \gamma_{\frac{1+2 m}{2(m+1)}}^{(j)}\right)^{\frac{s}{2(m+1)}}\right] \mathcal{M}(s)
$$

where the $\gamma_{a_{j}}^{(j)}$ denote independent gamma variables with respective parameters $a_{j}$.

Now, from the beta-gamma algebra, we deduce, for any $j \leq m-1$ :

$$
\gamma_{\frac{1+2 j}{2(m+1)}}^{(j)} \stackrel{(\text { law })}{=} \gamma_{\frac{1+2 m}{2(m+1)}}^{(j)} \beta\left(\frac{1+2 j}{2(m+1)}, \frac{m-j}{m+1}\right) .
$$

Thus, we obtain, again by comparing (12) and (13):

$$
\mathcal{M}(s)=(m+1)^{s / 2} \mathbb{E}\left[\left(\prod_{j=0}^{m-1} \beta\left(\frac{1+2 j}{2(m+1)}, \frac{m-j}{m+1}\right)\right)^{\frac{s}{2(m+1)}}\right]
$$

which entails:

$$
\mathbb{E}\left[Z_{m}^{s}\right]=(m+1)^{s / 2} \mathbb{E}\left[\left(\prod_{j=0}^{m-1} \beta\left(\frac{1+2 j}{2(m+1)}, \frac{m-j}{m+1}\right)\right)^{\frac{s}{2(m+1)}}\right]
$$

that is, equivalently,

$$
Z_{m} \stackrel{(\text { law })}{=}(m+1)^{1 / 2}\left(\prod_{j=0}^{m-1} \beta\left(\frac{1+2 j}{2(m+1)}, \frac{m-j}{m+1}\right)\right)^{\frac{1}{2(m+1)}}
$$

\section{Some remarks about Theorem 1}

\subsection{A further extension}

We tried to extend Theorem 1 by taking a product of independent martingales $X^{(i)}$, solution of $(2)$ with different $m_{i}$ 's. Here are the details of our attempt. We are looking for the existence of a variable $Z$ such that the martingale

$$
M(t)=\left(\prod_{j=0}^{p-1} X_{t}^{\left(m_{j}\right)}\right) Z
$$


satisfies the properties i) and ii). Here $p,\left(m_{j}\right)_{0 \leq j \leq p-1}$ are integers and $X^{\left(m_{j}\right)}$ is the solution of the EDS (2) associated to $m_{j}$, the martingales being independent for $j$ varying. In order that $M$ enjoys the Brownian scaling property, we need the following relation

$$
\sum_{j=0}^{p-1} \frac{1}{m_{j}+1}=1
$$

Following the previous computations, see (6), the Mellin transform $\mathcal{M}(s)$ of $Z$ should satisfy

$$
\frac{\Gamma\left(\frac{1+s}{2}\right)}{\Gamma\left(\frac{1}{2}\right)}=\left(\prod_{j=0}^{p-1} \frac{\Gamma\left(\frac{2 m_{j}+1+s}{2\left(m_{j}+1\right)}\right)}{\Gamma\left(\frac{2 m_{j}+1}{2\left(m_{j}+1\right)}\right)}\right) \mathcal{M}(s) .
$$

We recall (see (9)) the Gauss multiplication formula

$$
\frac{\Gamma\left(\frac{1+s}{2}\right)}{\sqrt{\pi}}=p^{s / 2} \prod_{j=0}^{p-1}\left(\frac{\Gamma\left(\frac{1+s+2 j}{2 p}\right)}{\Gamma\left(\frac{1+2 j}{2 p}\right)}\right)
$$

To find $\mathcal{M}(s)$ from (15), (16), we give some probabilistic interpretation:

$$
\frac{\Gamma\left(\frac{1+s+2 j}{2 p}\right)}{\Gamma\left(\frac{1+2 j}{2 p}\right)}=\mathbb{E}\left[\gamma_{(1+2 j) / 2 p}^{s / 2 p}\right]
$$

whereas

$$
\frac{\Gamma\left(\frac{2 m_{j}+1+s}{2\left(m_{j}+1\right)}\right)}{\Gamma\left(\frac{2 m_{j}+1}{2\left(m_{j}+1\right)}\right)}=\mathbb{E}\left[\gamma_{\left(1+2 m_{j}\right) / 2\left(m_{j}+1\right)}^{s / 2\left(m_{j}+1\right)}\right] .
$$

Thus, we would like to factorize

$$
\gamma_{(1+2 j) / 2 p}^{1 / 2 p} \stackrel{(\text { law })}{=} \gamma_{\left(1+2 m_{j}\right) / 2\left(m_{j}+1\right)}^{1 / 2\left(m_{j}+1\right)} z_{m_{j}, p}^{(j)}
$$

for some variable $z_{m_{j}, p}^{(j)}$ to conclude that

$$
Z=p^{1 / 2} \prod_{j=0}^{p-1} z_{m_{j}, p}^{(j)} .
$$

It remains to find under which condition the identity (17) may be fulfilled. We write

$$
\gamma_{(1+2 j) / 2 p} \stackrel{(\text { law })}{=} \gamma_{\left(1+2 m_{j}\right) / 2\left(m_{j}+1\right)}^{p /\left(m_{j}+1\right)}\left(z_{m_{j}, p}^{(j)}\right)^{2 p} .
$$

Now, if $\frac{1+2 j}{2 p}<\frac{1+2 m_{j}}{2\left(m_{j}+1\right)}$, we may apply the beta-gamma algebra to obtain 


$$
\gamma_{(1+2 j) / 2 p} \stackrel{(\text { law })}{=} \gamma_{\left(1+2 m_{j}\right) / 2\left(m_{j}+1\right)} \beta\left(\frac{1+2 j}{2 p}, \frac{1+2 m_{j}}{2\left(m_{j}+1\right)}-\frac{1+2 j}{2 p}\right)
$$

but in (18), we need to have on the right-hand side $\gamma_{\left(1+2 m_{j}\right) / 2\left(m_{j}+1\right)}^{p /\left(m_{j}+1\right)}$ instead of $\gamma_{\left(1+2 m_{j}\right) / 2\left(m_{j}+1\right)}$.

However, it is known that

$$
\gamma_{a} \stackrel{(\text { law })}{=} \gamma_{a}^{c} \gamma_{a, c}
$$

for some variable $\gamma_{a, c}$ independent of $\gamma_{a}$ for any $c \in(0,1]$. This follows from the self-decomposable character of $\ln \left(\gamma_{a}\right)$. Thus, we seem to need $\frac{p}{m_{j}+1} \leq 1$. But, this condition is not compatible with (14) unless $m_{j}=m=p-1$.

\subsection{Asymptotic study}

We study the behavior of the product $X_{1}^{(1)} \ldots X_{1}^{(m+1)}$, resp. $Z_{m}$, appearing in the right-hand side of the equality in law (5), when $m \longrightarrow \infty$. Recall from (4) that

$$
\left|X_{1}\right| \stackrel{(\operatorname{law})}{=}\left(2 \gamma_{\frac{2 m+1}{2(m+1)}}\right)^{\frac{1}{2(m+1)}}
$$

We are thus led to consider the product

$$
\Theta_{a, b, c}^{(p)}=\left(\prod_{i=1}^{p} \gamma_{a-b / p}^{(i)}\right)^{c / p}
$$

where in our set up of Theorem $1, p=m+1, a=1, b=c=1 / 2$.

$$
\begin{aligned}
\mathbb{E}\left[\left(\Theta_{a, b, c}^{(p)}\right)^{s}\right] & =\prod_{i=1}^{p} \mathbb{E}\left[\left(\gamma_{a-b / p}^{(i)}\right)^{c s / p}\right] \\
& =\left(\frac{\Gamma\left(a-\frac{b}{p}+\frac{c s}{p}\right)}{\Gamma\left(a-\frac{b}{p}\right)}\right)^{p} \\
& =\exp \left[p\left(\ln \left(\Gamma\left(a+\frac{c s-b}{p}\right)\right)-\ln \left(\Gamma\left(a-\frac{b}{p}\right)\right)\right)\right] \\
& \longrightarrow \exp \left(\frac{\Gamma^{\prime}(a)}{\Gamma(a)} c s\right) .
\end{aligned}
$$

Thus, it follows that

$$
\Theta_{a, b, c}^{(p)} \underset{p \rightarrow \infty}{\stackrel{\mathbb{P}}{\longrightarrow}} \exp \left(\frac{\Gamma^{\prime}(a)}{\Gamma(a)} c\right)
$$

implying that

$$
\left|X_{1}^{(1)} \ldots X_{1}^{(m+1)}\right| \underset{p \rightarrow \infty}{\stackrel{\mathbb{P}}{\longrightarrow}} \exp (-\gamma / 2)
$$

and 


$$
\exp (-\gamma / 2) Z_{m} \underset{m \rightarrow \infty}{\stackrel{(\text { law })}{\longrightarrow}}|N|
$$

where $\gamma=-\Gamma^{\prime}(1)$ is the Euler constant.

We now look for a central limit theorem for $\Theta_{a, b, c}^{(p)}$. We consider the limiting distribution of

$$
\begin{gathered}
\sqrt{p}\left\{\frac{c}{p} \sum_{i=1}^{p} \ln \left(\gamma_{a-b / p}^{(i)}\right)-c \frac{\Gamma^{\prime}(a)}{\Gamma(a)}\right\} \\
\mathbb{E}\left(\exp \left[c s \sqrt{p}\left\{\frac{1}{p} \sum_{i=1}^{p} \ln \left(\gamma_{a-b / p}^{(i)}\right)-\frac{\Gamma^{\prime}(a)}{\Gamma(a)}\right\}\right]\right) \\
=\mathbb{E}\left[\prod_{i=1}^{p}\left(\gamma_{a-b / p}^{(i)}\right)^{c s / \sqrt{p}}\right] \exp \left(-c s \sqrt{p} \frac{\Gamma^{\prime}(a)}{\Gamma(a)}\right) \\
=\mathbb{E}\left[\left(\gamma_{a-b / p}^{(i)}\right)^{c s / \sqrt{p}}\right]^{p} \exp \left(-c s \sqrt{p} \frac{\Gamma^{\prime}(a)}{\Gamma(a)}\right) \\
=\left(\frac{\Gamma\left(a-\frac{b}{p}+\frac{c s}{\sqrt{p}}\right)}{\Gamma\left(a-\frac{b}{p}\right)}\right)^{p} \exp \left(-c s \sqrt{p} \frac{\Gamma^{\prime}(a)}{\Gamma(a)}\right) \\
=\exp \left[p\left(\ln \left(\Gamma\left(a-\frac{b}{p}+\frac{c s}{\sqrt{p}}\right)\right)-\ln \left(\Gamma\left(a-\frac{b}{p}\right)\right)\right)-c s \sqrt{p} \frac{\Gamma^{\prime}(a)}{\Gamma(a)}\right] \\
=\exp \left(\frac{c^{2} s^{2}}{2}(\ln (\Gamma))^{\prime \prime}(a)+O\left(m^{-1 / 2}\right)\right)
\end{gathered}
$$

We thus obtain that

$$
\sqrt{p}\left\{\frac{c}{m} \sum_{i=1}^{m} \ln \left(\gamma_{a-b / m}^{(i)}\right)-c \frac{\Gamma^{\prime}(a)}{\Gamma(a)}\right\} \stackrel{(\text { law })}{\longrightarrow} N\left(0, \sigma^{2}\right)
$$

where $N\left(0, \sigma^{2}\right)$ denotes a centered Gaussian variable with variance:

$$
\sigma^{2}=c^{2}(\ln (\Gamma))^{\prime \prime}(a)=c^{2}\left[\frac{\Gamma^{\prime \prime}(a)}{\Gamma(a)}-\left(\frac{\Gamma^{\prime}(a)}{\Gamma(a)}\right)^{2}\right] .
$$

or, equivalently

$$
\left(\Theta_{a, b, c}^{(p)} \exp \left(\frac{\Gamma^{\prime}(a)}{\Gamma(a)} c\right)\right)^{\sqrt{p}} \underset{p \rightarrow \infty}{\stackrel{(\text { law })}{\longrightarrow}} \exp \left(N\left(0, c^{2}(\ln (\Gamma))^{\prime \prime}(a)\right)\right)
$$

\section{References}

1. Albin, J. M. P. A continuous non-Brownian motion martingale with Brownian motion marginal distributions. Statist. Probab. Lett. 78 (2008), no. 6, 682-686. 
2. Andrews, G.; Askey, R. and Roy, R. Special functions. Encyclopedia of Mathematics and its Applications, 71. Cambridge University Press, Cambridge, 1999.

3. Chaumont, L. and Yor, M. Exercises in probability. A guided tour from measure theory to random processes, via conditioning. Cambridge Series in Statistical and Probabilistic Mathematics, 13. Cambridge University Press, Cambridge, 2003.

4. Hamza, K. and Klebaner, F. A family of non-Gaussian martingales with Gaussian marginals. J. Appl. Math. Stoch. Anal. (2007), Art. ID 92723, 19 pp.

5. Madan, D. and Yor, M. Making Markov martingales meet marginals: with explicit constructions. Bernoulli 8 (2002), no. 4, 509-536. 